\title{
Verifying the Sleep-Inducing Effect of a Mother's Rocking Motion in Adults
}

\author{
Hiroaki Shibagaki \\ Department of Computer Science and Engineering, Graduate School of Engineering, Nagoya Institute of Technology \\ Gokiso-cho, Syouwa-ku, Nagoya, Aichi 466-8555, Japan \\ Keishi Ashida \\ Department of Computer Science and Engineering, Graduate School of Engineering, Nagoya Institute of Technology \\ Gokiso-cho, Syouwa-ku, Nagoya, Aichi 466-8555, Japan

\section{Yoshifumi Morita} \\ Department of Electrical and Mechanical Engineering, Graduate School of Engineering, Nagoya Institute of Technology \\ Gokiso-cho, Syouwa-ku, Nagoya, Aichi 466-8555, Japan

\section{Ryojun Ikeura} \\ Division of Mechanical Engineering, Graduate School of Engineering, Mie University \\ 1577 Kurimamachiyacho, Tsu, Mie 514-8507, Japan \\ Kiyoko Yokoyama \\ Graduate School of Design and Architecture, Nagoya City University \\ 2-1-10 Kitachikusa, Chikusa-ku, Nagoya, Aichi 464-0083, Japan \\ E-mail: h.shibagaki.502@nitech.jp
}

\begin{abstract}
Previously, we concluded that a mother's rocking motion is the most effective motion for inducing sleep in adults. We call it the candidate rocking motion. In this study, we confirm that the candidate rocking motion is more effective in inducing sleep than using no rocking motion. Moreover, we find that different to aromatherapy, the effectiveness of the candidate rocking motion varies only slightly between individuals. We conclude that the candidate rocking motion is effective for inducing sleep in adults.
\end{abstract}

Keywords: rocking motion, mother's rocking motion, sleep-inducing effect, electroencephalogram analysis

\section{Introduction}

Recently, the number of people suffering from high levels of stress has increased. This phenomenon is called a stressful society. ${ }^{1}$ Every year, an increasing number of people suffer from stress-related illnesses. Moreover, sleep disorders are becoming more widespread in modern society because stress is one of the causes of insomnia. Many research results related to sleep-inducing methods and relaxation methods have been reported. ${ }^{2-8}$

Robot for Interactive Body Assistance (RIBA) has been developed, which assists caregivers in lifting patients in and out of their beds and wheelchairs. ${ }^{9}$ For the caregivers in nursing homes, it is a large burden to ensure the wellbeing of the patients who suffer from stress-related illnesses and sleep disorders. ${ }^{10}$ The aim of our project is to develop a rocking motion that reduces 
stress and induces sleep, which will be installed in the RIBA. It is known that when a mother embraces and rocks her baby, the baby feels comfortable and falls asleep quickly. ${ }^{11,12} \mathrm{We}$ assume that a rocking motion simulating a mother's embrace would have the same effect on adults. We expect that our research results will be useful for other relaxation systems and robots.

In our previous study, we analyzed a mother's embrace and rocking motion, and we designed and constructed an excitation apparatus that simulates a mother's rocking motion. We found that a mother uses two types of rocking motions and constructed a model ${ }^{13}$ Moreover, we examined ten types of rocking motions and found that the linear motion component of a mother's rocking motion was the most effective rocking motion for inducing sleep in adults. We refer to this motion as the candidate rocking motion. ${ }^{14}$ However, we did not compare the sleep-inducing effect of the candidate rocking motion with other sleep-inducing methods, such as aromatherapy or music. ${ }^{2,3,4}$

In this paper, we report on our comparison of the sleep-inducing effect on adults of the candidate rocking motion, no stimulus, and aromatherapy. We evaluated the sleep-inducing effect through an electroencephalogram analysis.

\section{Excitation Apparatus Simulating a Mother's Rocking Motion}

In our previous work ${ }^{13}$, we analyzed a mother's embrace and rocking motion to identify the motion's features. Based on the analysis results, we found that there are two types of motions, both consisting of linear motion and rotational motion and created a model of a mother's rocking motion, which is represented by

$\left\{\begin{array}{l}x^{r e f}(t)=A_{x} \sin \left(2 \pi f_{x} t\right), \\ \theta^{\text {eff }}(t)=A_{\theta} \sin \left(2 \pi f_{\theta} t+\phi\right)+B_{\theta}\end{array}\right.$,

where $x^{r e f}(t)$ is the position and $\theta^{\text {ref }}(t)$ is the angle of the center of gravity of the baby. $B_{\theta}$ was determined from the angle of a comfortable sitting position reported in Ref. 15. We identified the rocking vibrations using the excitation apparatus shown in Fig. 1. The excitation apparatus has two degrees of freedom for linear motion and rotational motion.

In our previous work $^{14}$, from subjective experimental results, we found that, of the ten types of rocking motions examined, the linear motion component of a mother's rocking motion was the most

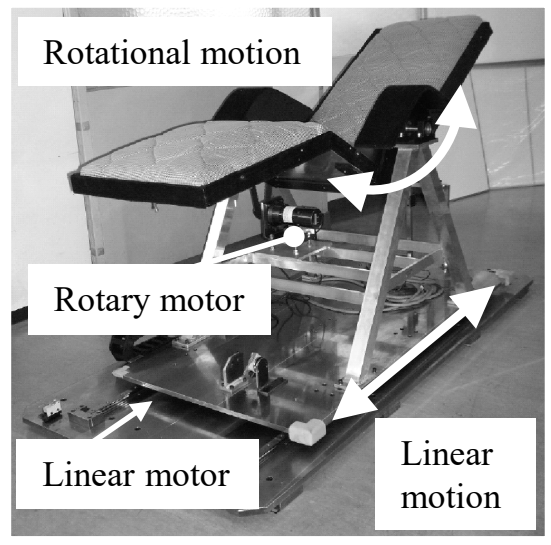

Fig. 1. Photograph of excitation apparatus.

effective rocking motion for inducing sleep in adults. The parameters of the candidate rocking motion were determined as $A_{x}=0.098[\mathrm{~m}], f_{x}=0.234[\mathrm{~Hz}], A_{\theta}=0$ [deg], $f_{\theta}=0[\mathrm{~Hz}], \phi=\pi / 2[\mathrm{rad}]$, and $B_{\theta}=25[\mathrm{rad}]$.

\section{Evaluation Method}

\subsection{Electroencephalogram analysis and sleep stage}

We computed the sleep stage through an electroencephalogram (EEG) analysis. We used the SYNA ACT MT1 1 system (NEC Medical Systems Ltd.) to measure the brain waves and the MATLAB computing environment (The MathWorks, Inc.) to conduct a frequency analysis. We placed the EEG electrodes according to the International 10-20 system. To compute the sleep stage, we used the quantitative evaluation method ${ }^{16}$ that was developed based on the definition by the Japanese Society of Sleep Research. We differentiate four different sleep stages. Sleep stage $\mathrm{W}$ indicates the state when being awake with mental activity, sleep stage 1 indicates the awakening and resting state, sleep stage 2 indicates a light sleeping state, and sleep stage 3 indicates a deep sleeping state.

\subsection{Evaluation index of sleep-inducing effect}

We evaluated the sleep-inducing effect with focus on "sleep latency" and "sleep depth." We define "sleep latency" as the time until the sleep enters stage 2 and denote it as $t_{\min }$. We define "sleep depth" as the total time spent in sleep stage 2 and sleep stage 3 , and we denote it as $S_{\text {sum. }}$. 


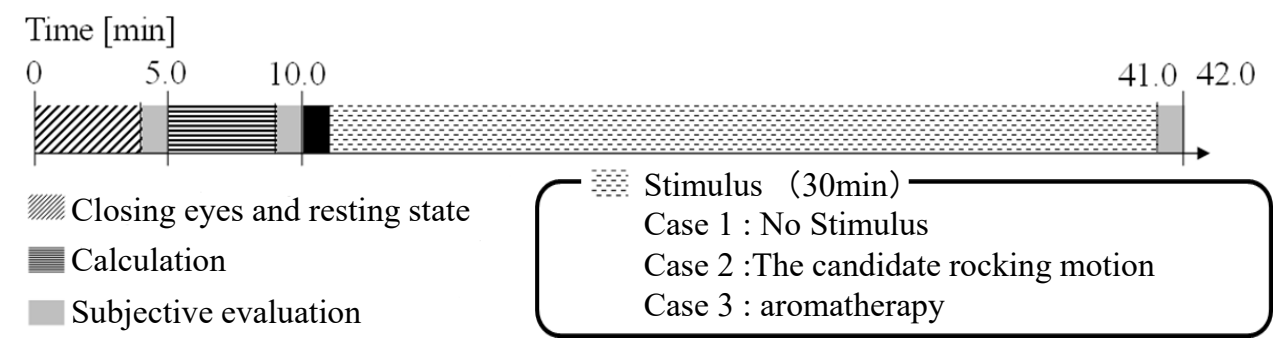

Closing eyes and resting state (Preparation to Provide stimulus)

Fig. 2. Experimental protocol.

\section{Experiment}

\subsection{Experimental method}

Fig. 2 shows the experimental protocol. Each experiment took 42 minutes. For each test subject, we conducted three experiments in the morning under different conditions and on different dates. The start times are the same for each test subject. To conduct a comparative study, we prepared three different conditions, namely without stimulus, with the candidate rocking motion, and with aromatherapy. We refer to them as Case 1, Case 2, and Case 3, respectively. For the aromatherapy, we picked genuine lavender (SANOFLORE) because of its verified relaxing effect. $^{17}$

\subsection{Experimental results and consideration}

The test subjects consisted of ten healthy males. The mean value and standard deviation of their ages were $21.8 \pm 0.6$. We conducted 30 experiments with a mean room temperature and standard deviation of $23.1 \pm$ $2.3{ }^{\circ} \mathrm{C}$, a mean humidity and standard deviation of 26.8 $\pm 5.6 \%$, and a mean illuminance and standard deviation of $22.3 \pm 14.91 \mathrm{x}$.

\subsubsection{Sleep stage}

We computed the sleep stage from an electroencephalogram (EEG) power spectrum for each analysis sections (AS). We had nine AS of sleep stages. The time of each AS was uniformed in 4 minutes. Fig. 3 shows the sleep stages of Subject D as an example. AS No. 1 refers to closed eyes and resting state before calculation. AS No. 2 refers to calculation. AS No. 3 to AS No. 9 refer to stimulus, namely either Case 1, Case 2,

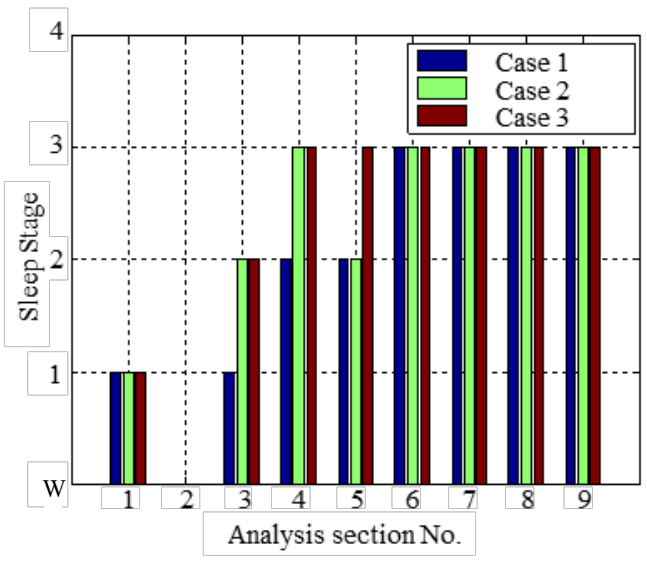

Fig. 3. Sleep stages of Subject D in three cases.

or Case 3. We compared Case 2 with Case 1 and Case 3 in AS No. 3 to AS No. 9.

On the other hand, it was reported that there are individual differences in aromatherapy. ${ }^{18}$ We had three test subjects for whom $t_{\text {min }}$ was larger or $S_{\text {sum }}$ was smaller in at least three sections of Case 3 as compared to Case 1. We can conclude that the sleep induction of the three test subjects became worse by applying aromatherapy. Therefore, we prepared two test groups, Group A and Group B. Group A consisted of all ten subjects, while Group B did not contain the three test subjects mentioned above and consisted only of the seven remaining subjects. For Group B, we can compare the sleep-inducing effect of the candidate rocking motion with that of aromatherapy under severe conditions.

Figs. 4 and 5 show the mean values and standard deviations of the sleep stages of Group A and Group B, respectively. Most of the mean values of the sleep 


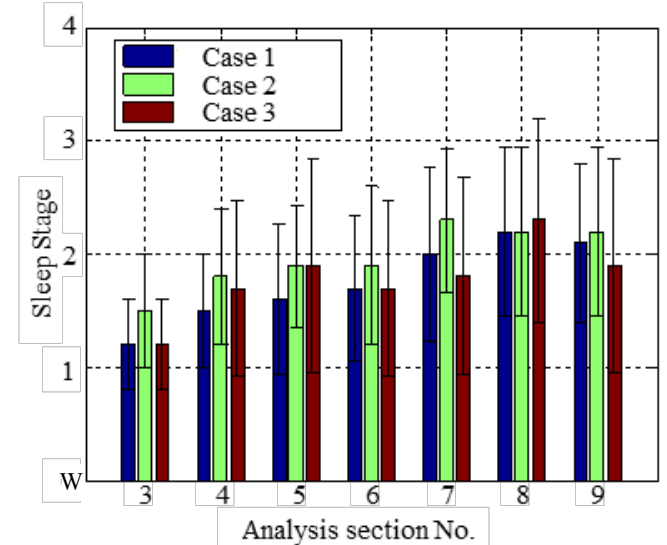

Fig. 4. Mean values and standard deviations of sleep stages in Group A.

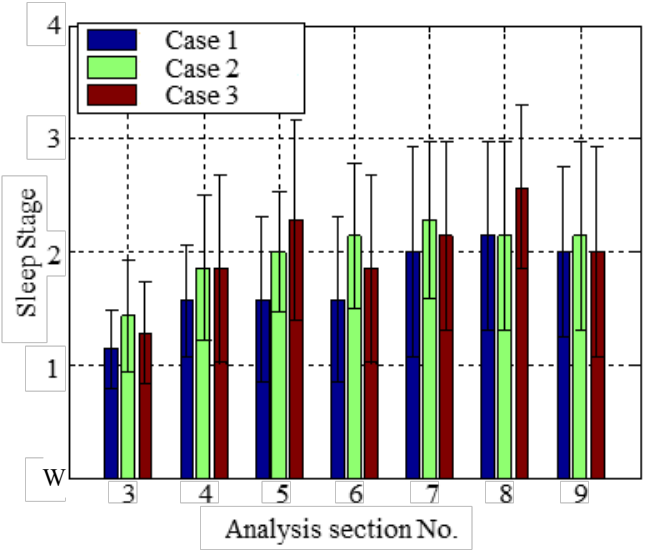

Fig. 5. Mean values and standard deviations of sleep stages in Group B.

stages in Case 2 were larger than the mean values in Case 1. In Fig. 5, most of the mean values of the sleep stages in Case 3 were larger than the mean values in Case 1. In Fig. 4, however, most of the mean values of the sleep stages in Case 3 were equal to or smaller than the mean values in Case 1 from AS No. 6 to AS No. 9.

\subsubsection{Evaluation of sleep-inducing effect}

We evaluated the sleep-inducing effect using the evaluation indices $t_{\min }$ and $S_{\text {sum }}$. The mean values and standard deviations of $t_{\min }$ and $S_{\text {sum }}$ of Group A and Group B are shown in Figs. 6 and 7, respectively.

Figs. 6 and 7 show that the mean values of $t_{\min }$ and $S_{\text {sum }}$ in Case 2 were equal to or greater than those in Case 3. We performed a statistical analysis using the IBM SPSS Statistics 22 software package. First, we verified the normal distribution by a Shapiro-Wilk test

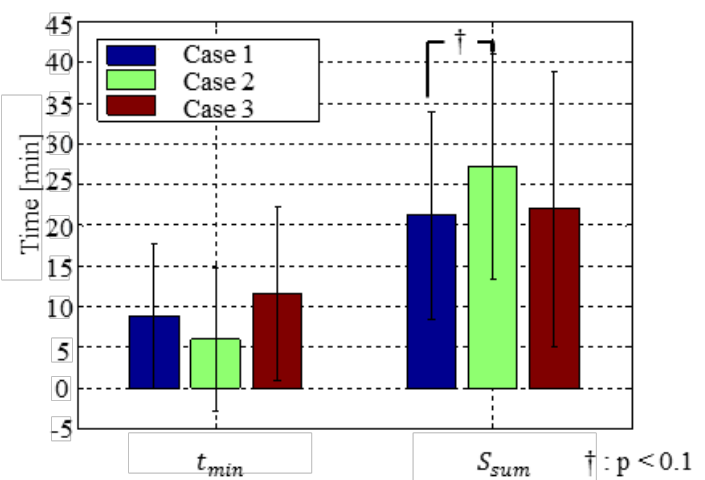

Fig. 6. Mean values and standard deviations of the two performance indices in Group A.

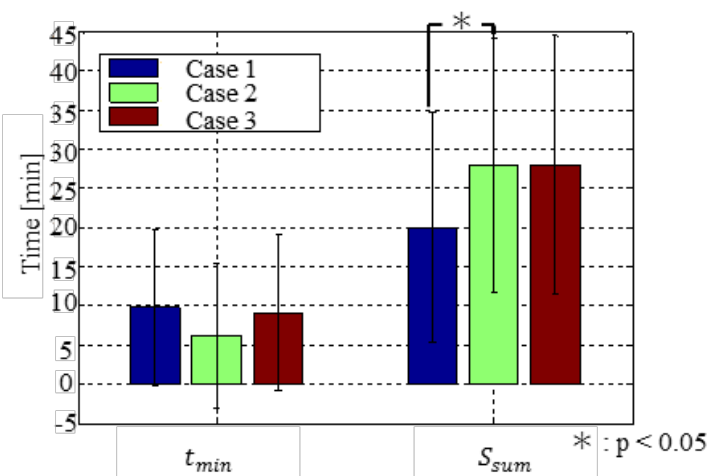

Fig. 7. Mean values and standard deviations of the two performance indices in Group B.

and the homoscedasticity by a Levene test. The distribution of $t_{\min }$ did not exhibit a normal distribution and showed homoscedasticity in both groups. The distribution of $S_{\text {sum }}$ exhibited a normal distribution and showed homoscedasticity in both groups. Thus, we performed a one-way variance analysis and applied the Bonferroni method on $S_{\text {sum }}$ of both groups. We also performed the Friedman test and the Wilcoxon signed rank sum test adjusted by the Bonferroni method on $t_{\min }$ of both groups.

We observed a significant trend $(p=0.055)$ between the values of the $S_{\text {sum }}$ index in Case 1 and Case 2 of Group A and significant differences $(p=0.040)$ between the values in Case 1 and Case 2 of Group B.

\subsubsection{Consideration}

Fig. 6 shows that in Group A, Case 2 has the smallest mean value of $t_{\min }$. However, there were no significant differences between any two pairs of all the cases. Fig. 7 shows the same results for Group B. However, Fig. 6 and Fig. 7 show that Case 2 has the largest mean value 
of $S_{\text {sum }}$ for both, Group A and Group B. There were no significant differences except between Case 1 and Case 2 .

Fig. 7 shows that the mean values of $S_{\text {sum }}$ in Case 2 and Case 3 are almost the same and that there is a significant difference between Case 1 and Case 2. However, there is no significant difference between Case 1 and Case 3. Therefore, we assume that, different to the candidate rocking motion, in the case of aromatherapy there are individual differences in the sleep-inducing effect.

\section{Conclusion}

In this paper, we demonstrated that the candidate rocking motion promotes a deep sleep in adults. We also showed that individual differences in the sleep-inducing effect were smaller in the case of the candidate rocking motion than in the case of aromatherapy.

As future work, we plan to compare the sleepinducing effect of the rocking motion with sleeping pills.

\section{References}

1. Ito, H., Ishino, Y., Kayukawa, Y, Tomita, S., Uchimura, N., and Murasaki, M., Adverse Effects of Sleep Deprivation, Iho Fuji, No.124 (2003), p.1. (in Japanese)

2. Yoshida, K., Mizuta, T., Takeshima, Y., Nishida, K., Tsukida K. and Takeda, C., The Effects of Foot Bathing on the Central Nervous System with or without Lavender Oil-An Electroencephalographic Study-, Fukui Medical University research magazine 2(1)(2) merger issue (2001). (in Japanese)

3. Momose, K., Fujisawa, Y. and Uchiyama, A., Effects of Music on Physiological Measurements and Mood States, and Their Correlation with Impression of the Music, Journal of International Society of Life Information Science, 22(2) (2004), pp.545-551. (in Japanese)

4. Kanazawa, Y., Moritani, K., Momose, I., Furuhashi, S., and Ohtsuka, Y., Examination of Stress Relieving Effects of Drinking Chamomile Tea and Peppermint Tea in the Morning, BULLETIN OF TENSHI COLLEGE, Vol.10 (2010), pp.23-34. (in Japanese)

5. Shimizu, K., Matsumoto, S., and Ohnuki, K., Sleep Promotion and Skin Condition Improvement Using (-)Bornyl Acetate, Cosmetology, Vol.24 (2016), pp.181189. (in Japanese)

6. Nagamatsu, T., Kitabatake, Y., and Sensui, H., Effect of Brief and Low-Intensity Stretch Exercise on Core Temperature, Stress, and Mood, Bulletin of the Physical Fitness Research Institute, No.110 (2012), pp.1-7. (in Japanese)

7. Han, J. and Uchiyama, A., The Effect of Odor Presentation on Immune Function after Stress Loading,
Journal Society Life Information Science, Vol.22 (2004), pp.574-579. (in Japanese)

8. Murase, C. and Kawamoto, R., The Effect of Aromatherapy (Scent of Lavender) on the Night Sleep of the Elderly [Translated from Japanese.], The Japanese journal of clinical nursing, Vol.32 (2006), pp.64-68. (in Japanese)

9. RIKEN-TRI Collaboration Center for Human-Interactive Robot Research (RTC), World's first robot that can lift up a human in its arms, RIBA, (online), available from $<\mathrm{http}: / /$ rtc.nagoya.riken.jp/RIBA/index-e.html $>$, (accessed on 28 November, 2016)

10. Mishima, K., Sleep Problems in Dementia, Proceedings of the Annual Meeting of the Japanese Research Group on Senile Dementia, Vol.17 (2010) (in Japanese), pp.109113.

11. Nakanishi, R., Ayashi Behavior Excluding Mother's Ayashi Play: Behavior that a mother hugs and rocks a child [Translated from Japanese.], Japan Society of Research on Early Childhood Care and Education, Vol.48 (1995) (in Japanese) pp.850-851.

12. Nakanishi, R., Repetitive behavior in mother's 'Ayashi behavior': On "Ayashi Behavior" Excluding "Ayashi Play" [Translated from Japanese.], Japan Society of Research on Early Childhood Care and Education, Vol.49 (1996), pp.150-151. (in Japanese)

13. Morita, Y., Yamaguchi, K., Ashida, K., Ikeura, R., Yokoyama, K., Verification of Sleep-Inducing Effect by Excitation Apparatus Simulating Mother's Embrace and Rocking Motion, Proceedings of the 9th International Workshop on Robot Motion and Control (RoMoCo '13), (2013), pp.80-85.

14. Ashida, K., Morita, Y., Ikeura, R., Yokoyama, K., Ding, M., Mori, Y., Effective rocking motion for inducing sleep in adults - Verification of effect of mother's embrace and rocking motion -, Journal of Robotics, Networks and Artificial Life, 1(4) (2015), pp.285-290.

15. Sasaki, Y., Kawamoto, T. and Yamazaki, N., Development of an Experiment Bed for Searching Comfort Semi-Sitting Position, Japanese Journal of Ergonomics, 42(6) (2006), pp.373-380.

16. Ashida, K., Yamaguchi, K., Morita, Y., Sato T., Ikeura, R. and Yokoyama, K., Verification of Sleep Inducing Effect by Mother's Embracing and Rocking Motion Consideration by Sleep Stage Judgment Based on EEG Analys is -, Proceeding of the 2013 JSME Conference on Robotics and Mechatronics, 2A2-G05(1)-(4) (2013). (in Japanese)

17. Yurugi Y. and Suzuki T., A review of Lavender Fragrance and Neural Function, The Bulletin of Kansai University of Health Sciences, 6 (2012), pp.109-115. (in Japanese)

18. Miyajima, M., Moriya, K., and Agishi, Y., The effects of bathing with, lavender oil on relaxation as assessed from changes in skin temperature, JAPANESE JOURNAL OF BIOMETEROLOGY, 34(4) (1997), pp.139-146. (in Japanese) 